\title{
Small-Tip Fast Recovery Imaging Using Non-Slice-Selective Tailored Tip-Up Pulses and Radiofrequency-Spoiling
}

\author{
Jon-Fredrik Nielsen, ${ }^{1 *}$ Daehyun Yoon, ${ }^{2}$ Douglas C. Noll ${ }^{1}$
}

Small-tip fast recovery (STFR) imaging is a new steady-state imaging sequence that is a potential alternative to balanced steady-state free precession. Under ideal imaging conditions, STFR may provide comparable signal-to-noise ratio and image contrast as balanced steady-state free precession, but without signal variations due to resonance offset. STFR relies on a tailored "tip-up," or "fast recovery," radiofrequency pulse to align the spins with the longitudinal axis after each data readout segment. The design of the tip-up pulse is based on the acquisition of a separate off-resonance (BO) map. Unfortunately, the design of fast (a few ms) slice- or slab-selective radiofrequency pulses that accurately tailor the excitation pattern to the local BO inhomogeneity over the entire imaging volume remains a challenging and unsolved problem. We introduce a novel implementation of STFR imaging based on "non-slice-selective" tip-up pulses, which simplifies the radiofrequency pulse design problem significantly. Out-of-slice magnetization pathways are suppressed using radiofrequency-spoiling. Brain images obtained with this technique show excellent gray/white matter contrast, and point to the possibility of rapid steady-state $T_{2} / T_{1}$-weighted imaging with intrinsic suppression of cerebrospinal fluid, through-plane vessel signal, and off-resonance artifacts. In the future, we expect STFR imaging to benefit significantly from parallel excitation hardware and high-order gradient shim systems. Magn Reson Med 69:657-666, 2013. @ 2012 Wiley Periodicals, Inc.

Key words: steady-state free precession; banding artifacts; inflow suppression; CSF suppression

Small-tip fast recovery (STFR) imaging is a new steadystate imaging sequence that is a potential alternative to balanced steady-state free precession (bSSFP) (1-3). The central idea in STFR, as originally proposed, is to design a slice-selective tip-down excitation pulse which "prephases" the spins according to the local off-resonance frequency. After data readout, spins are tipped back toward the longitudinal axis, using a tip-up, or "recovery,” radiofrequency pulse. This pulse sequence resembles the "fast recovery" or "driven equilibrium" sequences proposed previously but does not require spin-echo refocusing pulses, and hence, it can deposit less RF energy. Under

\footnotetext{
${ }^{1}$ Department of Biomedical Engineering, University of Michigan, Ann Arbor, Michigan, USA.

${ }^{2}$ Department of Electrical Engineering and Computer Science, University of Michigan, Ann Arbor, Michigan, USA.

Grant sponsor: National Institutes of Health; Grant number: R01NS058576 and R21EB012674.

*Correspondence to: Jon-Fredrik Nielsen, Ph.D., 1067 B.I.R.B., 2360 Bonisteel Avenue, Ann Arbor, Ml 48109-2108. E-mail: jfnielse@umich.edu

Received 1 September 2011; revised 6 March 2012; accepted 16 March 2012. DOI 10.1002/mrm.24289

Published online 17 April 2012 in Wiley Online Library (wileyonlinelibrary.com).

(C) 2012 Wiley Periodicals, Inc.
}

ideal conditions, STFR imaging has many of the benefits of bSSFP such as high signal-to-noise ratio efficiency, good flow properties, and combined T2/T1 weighting (4), but does not suffer from signal variation due to resonance offset (e.g., banding artifacts). Therefore, STFR has the potential to become a universal replacement for bSSFP and may obviate the need for special artifact-reduction techniques such as phase-cycled imaging (5) and multiple repetition time (TR) sequences (6-9).

Unfortunately, the design of fast (a few ms) slice- or slabselective RF pulses that accurately tailor the excitation pattern to the local $B 0$ inhomogeneity over the entire imaging volume remains a challenging and unsolved problem. The purpose of this article is to introduce a novel implementation of STFR imaging based on "non-slice-selective" tip-up pulses, which simplifies the RF pulse design problem significantly. Specifically, for two-dimensional (2D) single-slice imaging, the dimensionality of the tip-up pulse is reduced from three to two. The proposed method relies on RF-spoiling to suppress out-of-slice transverse magnetization pathways created by the tip-up pulse. We present phantom measurements that show that (i) the tip-up pulse is successful in aligning the spins along the longitudinal axis, and (ii) STFR produces enhanced signal compared to conventional spoiled gradient-echo (SPGR) imaging. We also present the first in vivo images to be obtained using the STFR principle, which show good gray/white matter contrast similar to bSSFP. A simplified signal model is also presented, which is shown to provide a useful qualitative description of STFR imaging.

\section{THEORY}

The proposed imaging principle is illustrated in Fig. 1. Just as in bSSFP or SPGR, a simple slice-selective tip-down pulse $\alpha$ is first played out, and the signal is acquired during a free precession interval of duration $T_{\text {free }}$, during which the spin precesses in the transverse plane with an angle $\theta(x, y)=\omega(x, y) T_{\text {free }}$, where $\omega(x, y)=\gamma B 0$ is the local off-resonance. After data readout, spins within the desired imaging slice are tipped back toward the longitudinal axis (mz) by a spatially tailored tip-up pulse $\beta(x, y)$. Note that the tip-up pulse can in principle be designed for arbitrary phase accrual $\theta(x, y)$, provided that $\theta(x, y)$ is sufficiently smooth relative to the spatial frequency content of the tailored tip-up pulse. In particular, $\theta(x, y)$ need not be small. As the tip-up pulse is non-slice-selective, spins outside the desired imaging slice will be excited (Fig. 1c). This out-ofslice signal is removed using spoiling, i.e., by inserting a gradient spoiler $\mathrm{S}$ after the tip-up pulse, and cycling the $\mathrm{RF}$ phase, for example, using a linear phase increment of $117^{\circ} \times n$, where $n$ is the RF shot number (10). Note that 

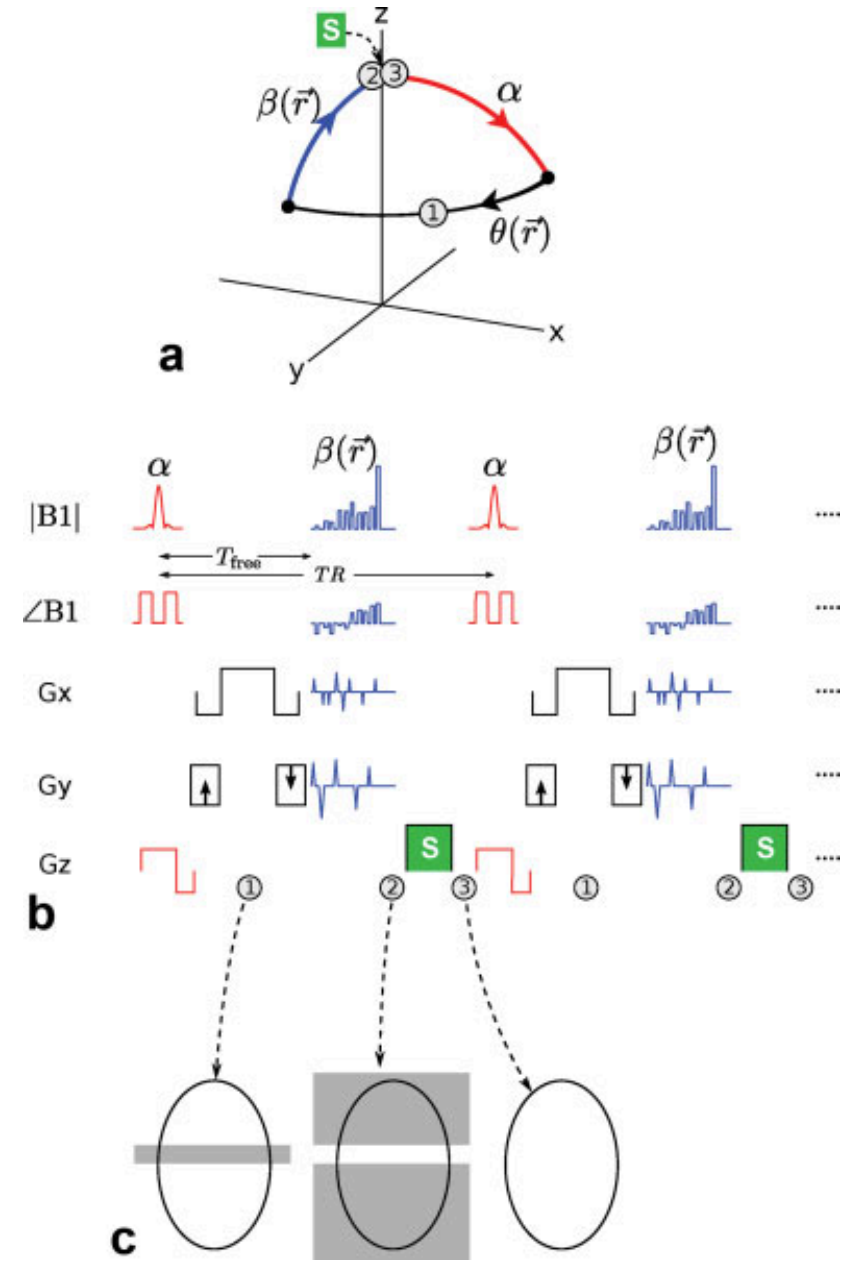

FIG. 1. Proposed STFR imaging principle. a:: Steady-state spin path for a single spin isochromat. b: Proposed STFR implementation, illustrative 2D imaging example. c: Desired transverse magnetization pattern at the time-points labeled " 1, " " 2 ," and " 3 " in (a) and (b), for an axial slice. At time-point " 1 ," only the imaging slice is excited and hence imaged (left). Immediately after the non-slice-selective tip-up pulse (2), spins within the imaging slice have been realigned with the z-axis, while out-of-slice spins have been excited (middle). After the gradient spoiler S (time-point 3), no transverse magnetization remains (right). TR-to-TR signal coherence pathways for out-of-slice spins are suppressed using RF-spoiling. [Color figure can be viewed in the online issue, which is available at wileyonlinelibrary.com.]

the tip-down and tip-up pulses have a common RF phase offset.

To make the distinction between bSSFP and STFR clear, Fig. 2 shows the steady-state spin paths in the $x z$-plane for a spin with zero off-resonance.

\section{Signal Theory Under Ideal Conditions}

To obtain a simple qualitative description of the STFR signal, we assume that the RF pulse duration is negligible. Clearly, this assumption is not met in general, because the RF pulse duration depends on the details of the tip-up pulse design. Nevertheless, we will show that the qualitative predictions based on this assumption are confirmed in vivo.
With this assumption, the steady-state transverse STFR signal $M_{\perp}$ immediately after the tip-down pulse can be shown to be

$$
\begin{aligned}
& M_{t}=M_{0} \\
& \times \frac{e^{-\frac{T_{\mathrm{s}}}{T_{1}}}\left(1-e^{-\frac{T_{\text {free }}}{T_{1}}}\right) \sin \alpha \cos \beta(x, y)+\left(1-e^{-\frac{T_{\mathrm{s}}}{T_{1}}}\right) \sin \alpha}{1-e^{-\frac{T_{\mathrm{s}}}{T_{1}}-\frac{T_{\text {free }}}{T_{2}}} \sin \alpha \sin \beta(x, y)-e^{-\frac{T_{\mathrm{s}}}{T_{1}}-\frac{T_{\text {free }}}{T_{1}}} \cos \alpha \cos \beta(x, y)}
\end{aligned}
$$

Here $T_{\mathrm{s}}$ is the delay needed for the gradient spoiler, which is played out immediately after the tip-up pulse. Equation 1 predicts that the STFR signal contains both $T_{1}$ and $T_{2}$ weighting. Furthermore, in the limits of TR $<<T_{2}$ and $T_{\mathrm{s}}=0$, and assuming $\beta=\alpha$, it can be shown that the STFR signal is equal to the bSSFP on-resonance signal with flip angle $2 \alpha$. Therefore, we expect STFR images to produce similar tissue contrast as bSSFP. Note that in the limits $\beta=0$ and $T_{\mathrm{s}}=0$, Eq. 1 is identical to the Ernst formula with flip angle $\alpha$. In addition, Eq. 1 predicts that the signal

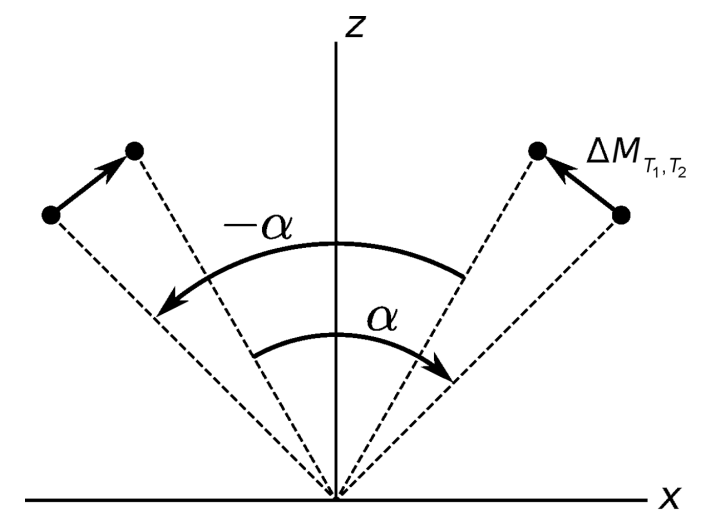

2

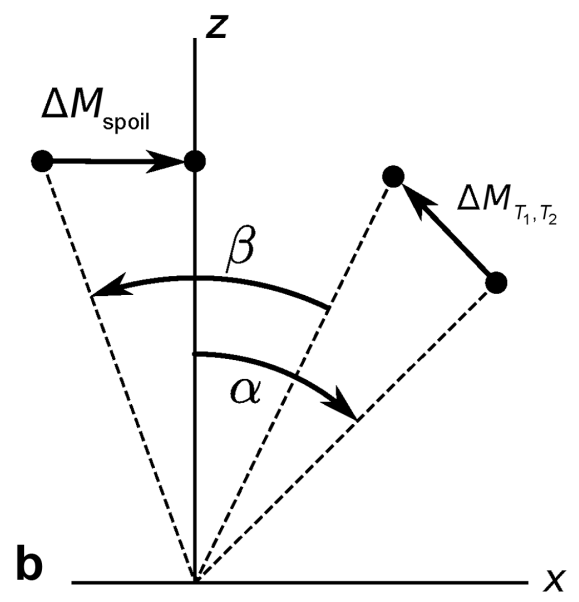

FIG. 2. Graphical illustration of the steady-state spin paths for (a) bSSFP and (b) the proposed STFR method, for an on-resonance spin (not drawn to scale). In bSSFP (a), the $T_{1}$ recovery and $T_{2}$ decay that occurs between RF pulses is perfectly balanced between alternating RF shots. In STFR (b), the $T_{1}$ and $T_{2}$ decay that occurs after the tipdown pulse $\alpha$, combined with the effect of the spoiler following the tip-up pulse $\beta$, act to bring the longitudinal magnetization back to the same starting point prior to each tip-down pulse. 

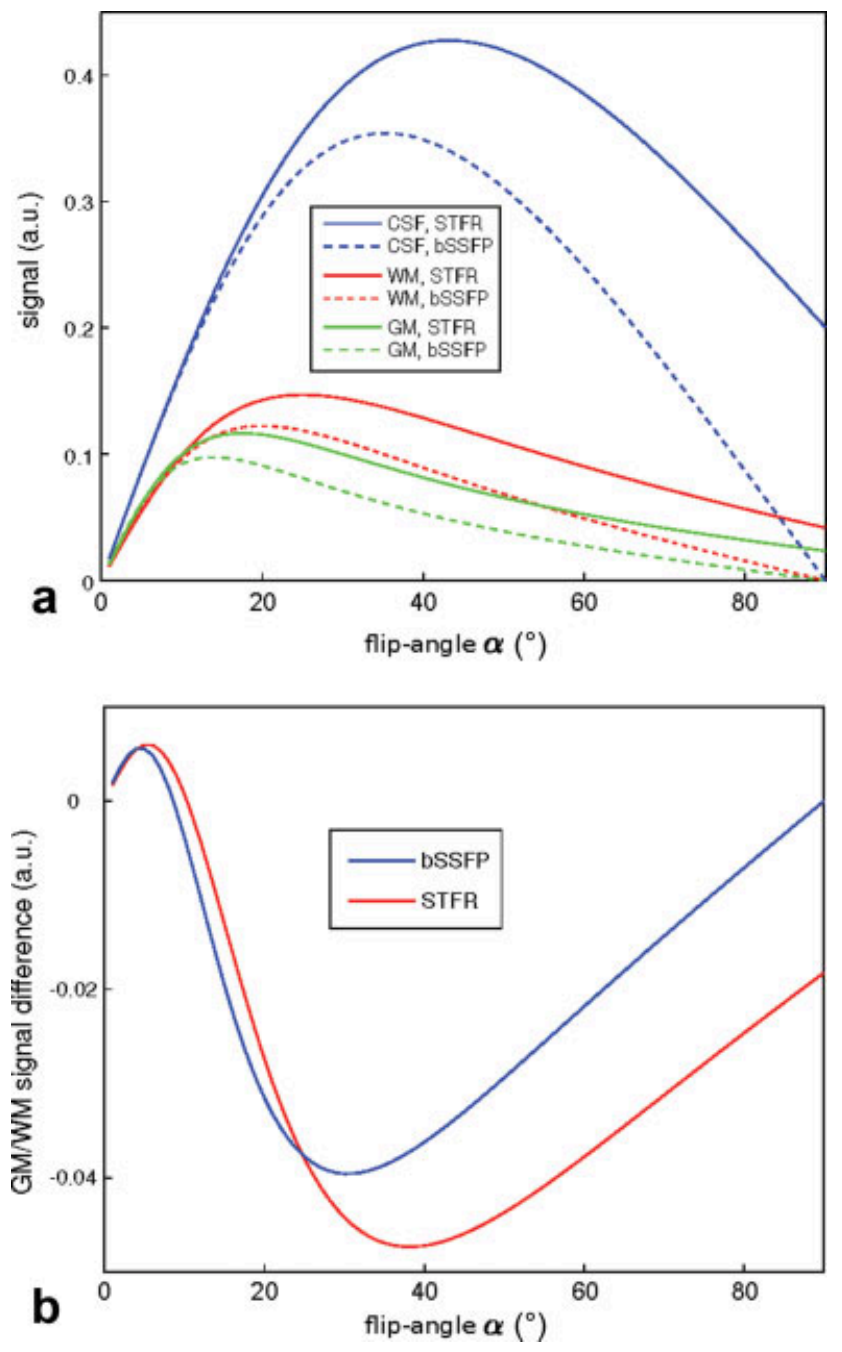

FIG. 3. Predicted tissue signal for STFR using Eq. $1\left(T_{\text {free }} / T R=\right.$ $8 / 12 \mathrm{~ms}, \alpha=\beta$ ), and for bSSFP. Note that the bSSFP curves were calculated using a flip angle of $2 \alpha$. a: Theory predicts that STFR produces high CSF signal for all flip angles, similar to bSSFP. b: Predicted gray/white matter contrast for STFR and bSSFP. STFR and bSSFP are expected to produce similar gray/white matter contrast. [Color figure can be viewed in the online issue, which is available at wileyonlinelibrary.com.]

from cerebrospinal fluid (CSF) is high when $\beta(x, y)=\alpha$, but drops off rapidly with decreasing $\beta(x, y)$ (due to the very long $T_{2}$ of CSF). This is illustrated in Fig. 9a, which shows the calculated signal for CSF and gray matter based on Eq. 1, assuming $T_{1} / T_{2}=4000 / 2000 \mathrm{~ms}$ and $1300 / 80 \mathrm{~ms}$ for CSF and gray matter, respectively. ${ }^{1}$ This suggests that hyperenhancement of CSF may be avoided by reducing $\beta(x, y)$ slightly.

Figure 3 shows the predicted signal for white matter, gray matter, and CSF, for flip angles $\alpha$ ranging from 0 to $90^{\circ}$. These plots show that STFR is expected to produce similar signal and tissue contrast as bSSFP.

${ }^{1}$ Reported $T_{1}$ and $T_{2}$ values for CSF vary, but they are generally in the range of several seconds $(11,12)$.

\section{Effect of an Imperfect Tip-Up Pulse}

In practice, the phase of the tip-up pulse will not match the local spin phase exactly, which will reduce the observed signal relative to the ideal theoretical value expressed in Eq. 1. We propose to use Eq. 1 to describe the STFR signal even in the case of such a phase mismatch, except that $T_{2}$ should be replaced by an "effective" transverse relaxation rate $T_{2_{\text {eff }}}$, defined by the relation $e^{-T_{\text {free }} / T_{2}} \cos (\Delta \theta) \equiv$ $e^{-T_{\text {free }} / T_{2} \text { eff }}$, or

$$
T_{2_{\text {eff }}} \equiv \frac{T_{2} T_{\text {free }}}{T_{\text {free }}-T_{2} \ln (\cos (\Delta \theta))} \quad(\Delta \theta \neq 0)
$$

where $\Delta \theta$ is the phase mismatch between the target phase $\theta(x, y)$ and the phase of the tip-up pulse. In essence, we are treating the phase mismatch $\Delta \theta$ as an additional " $T_{2}$ like" transverse relaxation factor. Specifically, we assume that the transverse magnetization vector component $M_{\perp}$ is reduced by a factor $e^{-T_{\text {free }} / T_{2}} \cos (\Delta \theta)$, as illustrated in Fig. 4.

\section{METHODS}

\section{Experimental Validation of Eqs. 1 and 2}

To validate Eqs. 1 and 2, we performed three imaging experiments in a uniform gelatin ball phantom. The pulse

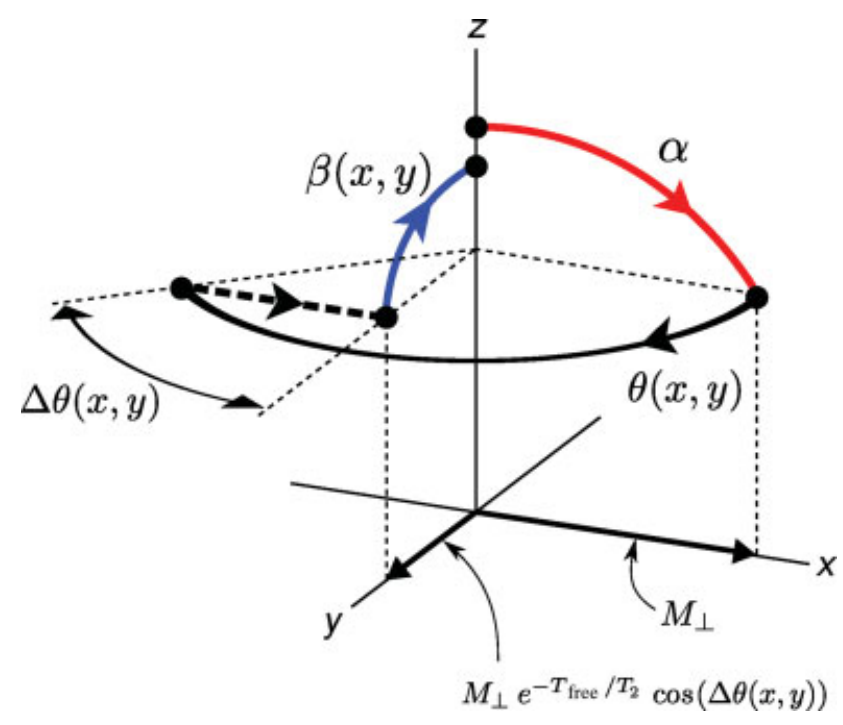

FIG. 4. Graphical illustration of the rationale behind Eq. 2. Immediately after the tip-down pulse $\alpha$, the transverse signal component has magnitude $M_{\perp}$ and is aligned with the axis of the tip-down pulse $(x)$. During the free precession interval, the transverse component is reduced to $M_{\perp} e^{-T_{\text {free }} / T_{2}}$ (not indicated in the figure). We then assume that there is a mismatch $\Delta \theta$ between the transverse spin phase and the phase of the tip-up pulse. As a result, only the component of $M_{\perp} e^{-T_{\text {free }} / T_{2}}$ along the axis of the tip-up pulse, i.e., $M_{\perp} e^{-T_{\text {free }} / T_{2}} \cos (\Delta \theta)$, is tipped back up. Equation 2 is obtained by defining an effective transverse relaxation rate $T_{2}$ defined by the relation $M_{\perp} e^{-T_{\text {free }} / T_{2}} \cos (\Delta \theta) \equiv M_{\perp} e^{-T_{\text {free }} / T_{2}}$ eff . [Color figure can be viewed in the online issue, which is available at wileyonlinelibrary.com.] 

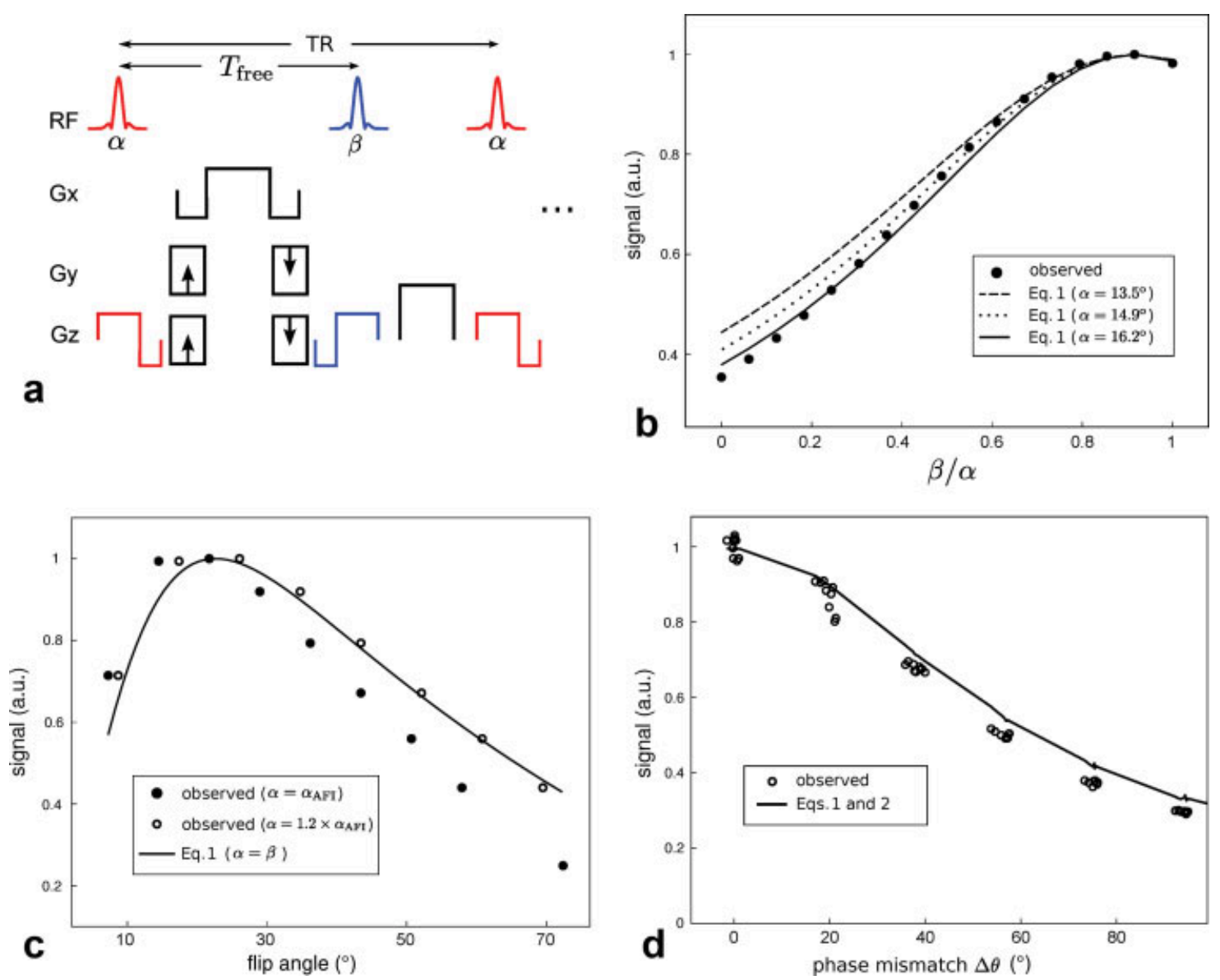

FIG. 5. Experimental validation of Eqs. 1 and 2. a: Pulse sequence diagram. The tip-up pulse is identical to the tip-down pulse, except time-reversed. b: Steady-state signal as a function of tip-up angle $\beta$, with the tip-down flip angle $\alpha$ held constant $\left(T_{\text {free }} / \mathrm{TR}=4.3 / 7.0 \mathrm{~ms}\right)$. c: Steady-state signal as a function of flip angle $\left(T_{\text {free }} / \mathrm{TR}=10.0 / 15.0 \mathrm{~ms}\right)$. d: Steady-state signal as a function of phase mismatch $\Delta \theta$ between the phase of the tip-up pulse and the spin phase $\left(T_{\text {free }} / \mathrm{TR}=9.3 / 12.0 \mathrm{~ms}\right)$. [Color figure can be viewed in the online issue, which is available at wileyonlinelibrary.com.]

sequence consisted of independently controlled tip-down and tip-up RF segments, and a conventional Cartesian (spin-warp) readout with balanced gradients (Fig. 5a). The tip-down pulse was a slice-selective sinc or ShinnarLe Roux (13) pulse with time-bandwidth product 4. The tip-up pulse was identical to the tip-down pulse, except time-reversed. The flip angle $\alpha$ was measured using actual flip-angle imaging (AFI) (14), with $\mathrm{TR}_{1} / \mathrm{TR}_{2}=10 / 110 \mathrm{~ms}$. The off-resonance map was measured using SPGR with $\mathrm{TR}=10 \mathrm{~ms}$ and echo times of 3 and $5 \mathrm{~ms}$. The acquisition bandwidth was $\pm 31.25 \mathrm{kHz} . T_{1}$ and $T_{2}$ were measured using inversion recovery and spin-echo, respectively $\left(T_{1} / T_{2}=520 / 50 \mathrm{~ms}\right)$.

The three different experiments were conducted on different days. In the first experiment, we varied the magnitude of the tip-up pulse $\beta$ from 0 to $\alpha$, with $\alpha$ held fixed. The middle slice from a $64 \times 64 \times 8$ threedimensional (3D) acquisition with field-of-view (FOV) $24 \times$ $24 \times 3 \mathrm{~cm}^{3}$ was used in the analysis. The tip-down pulse was of duration $0.5 \mathrm{~ms}$ (not including slice-select gradients) and slab thickness $10 \mathrm{~mm}$. In each resulting image, the mean signal within a region-of-interest with near-zero offresonance was calculated. The resulting signal curve was compared with the calculated values from Eq. $1\left(T_{\text {free }} / \mathrm{TR}=\right.$ $4.3 / 7.0 \mathrm{~ms} ; T_{1}=520 \mathrm{~ms}, T_{2_{\text {eff }}}=T_{2}=50 \mathrm{~ms}$ ).

In the second experiment, we varied the flip angle from approximately 10 to $70^{\circ}$, and recorded the mean signal within a region-of-interest with near-zero off-resonance. The resulting signal curve was compared with the calculated values from Eq. $1\left(T_{\text {free }} / \mathrm{TR}=10.0 / 15.0 \mathrm{~ms} ; T_{1}=\right.$ $520 \mathrm{~ms}, T_{2_{\text {eff }}}=T_{2}=50 \mathrm{~ms}$ ). The acquisition was $2 \mathrm{D}$ with $24 \times 24 \mathrm{~cm}^{2}$ FOV, $64 \times 64$ matrix, and a tip-down pulse of duration $2 \mathrm{~ms}$ and slice thickness $5 \mathrm{~mm}$.

In the third experiment, we introduced a linear offresonance across the phantom by adjusting the gradient shim. We then acquired one STFR image, with $\alpha=\beta$. The middle slice from a $64 \times 64 \times 83 \mathrm{D}$ acquisition with FOV $24 \times 24 \times 3 \mathrm{~cm}^{3}$ was used in the analysis. The same tipdown pulse as in the first experiment was used. We selected a region-of-interest consisting of a rectangular region near the center of the phantom, and for each pixel within this region-of-interest, the phase mismatch $\Delta \theta$, flip angle $\alpha$, and signal was recorded. The observed signal was plotted against $\Delta \theta$ and compared with the calculated values from Eq. 1 , with $T_{\text {free }} / \mathrm{TR}=9.3 / 12.0 \mathrm{~ms}, T_{1}=520 \mathrm{~ms}$, and $T_{2_{\text {eff }}}$ given by Eq. 2 . 


\section{D RF Pulse Design}

The tip-up pulse $\beta(x, y)$ is spatially tailored and hence requires multidimensional RF pulse design principles and/or a parallel transmit array. We consider in this work a small-tip design with two different excitation $k$-space trajectories: blipped and spiral $(15,16)$. A small-tip design is appropriate for brain imaging using the proposed method, because the optimal flip angle for gray matter is only $16^{\circ}$, one-half of the bSSFP optimal flip angle.

Blipped pulses, which consist of a train of weighted subpulses (e.g., slice-selective sincs), are relatively insensitive to gradient system imperfections such as unknown gradient delays and eddy currents. Therefore, blipped pulses are ideal for assessing the intrinsic image contrast of the proposed STFR sequence. We use the RF pulse design algorithm in Ref. 17, which uses a "greedy" search to obtain the pulse weights that best match the desired excitation pattern. In our previous implementation, each blip consisted of a slice-selective sinc pulse (a "spoke") of $\sim 0.5 \mathrm{~ms}$ duration. With the proposed implementation, the tip-up pulse is non-selective, and hence, the sinc pulses can be replaced with fast hard pulses. In this article, all tip-up pulses were implemented with 10 rectangular "blips," each lasting only $40 \mu \mathrm{s}$. We found empirically that this pulse train can achieve a maximum flip angle of just under $20^{\circ}$ without violating the peak B1 constraints of our system $(0.25 \mathrm{G})$. Note that no gradients are applied during RF transmission. The hard pulses are separated by $x$ and $y$ gradient "blips" that traverse excitation $k x-k y$ space. Note that this is different from an echo-planar trajectory, where time-varying RF and gradient fields are transmitted simultaneously. The total tip-up pulse duration, including all gradients, was 1.4$1.6 \mathrm{~ms}$. A corresponding pulse using slice-selective sinc pulses would require a pulse duration of $6.5 \mathrm{~ms}$.

Spiral tip-up pulses were designed using the small-tip design method in Ref. 18, implemented with the IRT Matlab toolbox (Jeff Fessler, University of Michigan). The advantage of spiral tip-up pulses is that they sample excitation $k$-space much more efficiently than blipped designs and are therefore expected to produce much better excitation accuracy (i.e., reduced $\Delta \theta$ ) for a given total RF pulse duration. The disadvantage of spirals is that they are very sensitive to system imperfections. As the exact gradient delays with respect to the RF waveform are generally not known, the spiral STFR acquisitions were repeated with different applied gradient delays. The RF waveform duration was $\sim 2.3 \mathrm{~ms}$, excluding the gradient prewinder.

In both designs, phase accrual during RF transmission was accounted for. We found in simulation that this was essential for accurate tip-up performance (not shown).

Note that we designed $\beta(x, y)$ by first creating an intermediate tip-down pulse using $e^{i \theta(x, y)}$ as the target excitation pattern, where

$$
\theta(x, y)=\omega(x, y) T_{\text {free }}
$$

is the expected spin precession angle based on the measured local off-resonance $\omega(x, y)$ in a $2 D$ slice. In the design of the intermediate tip-down pulse, the off-resonance was set to the negative of the observed B0 map. The resulting tip-down pulse was then used to obtain the final tip-up
Table 1

Summary of Phantom and Human Imaging Experiments

\begin{tabular}{lccl}
\hline & Tip-down angle $\alpha\left(^{\circ}\right)$ & Readout & Tip-up pulse \\
\hline Phantom & 8 & $3 D$ & Blipped \\
Session 1 & 8 & $3 D$ & Blipped \\
Session 2 & 8 & 3D & Spiral \\
Session 3 & $16 / 32$ & 2D & Spiral \\
\hline
\end{tabular}

pulse by traversing excitation $k$-space in reverse, effectively "undoing" the excitation created by the intermediate tip-down pulse. This procedure was the same for both blipped and spiral tip-up pulses.

\section{Phantom Imaging Experiments}

All imaging experiments were performed on a GE Signa 3T scanner using a commercial quadrature headcoil. A 3D readout was chosen to eliminate any possible influence of imperfect slice-profile on the final images. The tip-down pulse was a slice-selective sinc pulse of time-bandwidth product 4, duration $0.5 \mathrm{~ms}$ (not including slice-select gradients), and slab thickness $10 \mathrm{~mm}$. We measured the flip angle experimentally by acquiring multiple SPGR images at different TR in the phantom, and fitting to the Ernst formula (not shown). The observed tip-down angle of $\alpha=8^{\circ}$ was used, because it is approximately equal to the Ernst angle for brain tissue imaging at 3T, and should therefore give maximum SPGR signal. Note that this is less than the optimal flip angle for gray matter imaging using bSSFP and STFR at $3 \mathrm{~T}$, which is approximately $32^{\circ}$ and $16^{\circ}$, respectively. For the SPGR and STFR acquisitions, the gradient spoiler $S$ after the tip-up pulse consisted of a trapezoidal gradient along $z$. Off-resonance maps were acquired with a 3D SPGR sequence $(64 \times 64 \times 8$ matrix; FOV $24 \times 24 \times 3 \mathrm{~cm}$; flip angle $8^{\circ}$; TR $=10 \mathrm{~ms}$; and acquisition bandwidth $\pm 125 \mathrm{kHz}$ ), and only the central $2 \mathrm{D}$ slice was used in the design of the tailored tip-up pulse.

Phantom observations were made in the same uniform gelatin ball phantom used in the theory validation experiments $\left(T_{\text {free }}=5.7 \mathrm{~ms} ; 64 \times 64 \times 8\right.$ matrix; FOV $24 \times 24 \times 3 \mathrm{~cm}$; tip-down angle $\alpha=8^{\circ}$; TR/echo time $=9 / 2 \mathrm{~ms}$; acquisition bandwidth $\pm 125 \mathrm{kHz}$ ). We imaged the residual transverse magnetization after the tip-up pulse, to verify that the tip-up pulse brings the spins within the desired imaging slice back toward the longitudinal axis. We also performed SPGR, bSSFP, and STFR acquisitions with the same tipdown angle $\alpha$, to verify that STFR produces enhanced signal compared with SPGR.

\section{Human Volunteer Imaging}

One healthy human volunteer was imaged in three different sessions using the same pulse sequence as in the phantom imaging experiments (Table 1). In each session, we acquired SPGR, bSSFP, and STFR images, to compare image contrast. Both blipped (session 1) and spiral (sessions 2 and 3) tipup pulses were tested, using both $3 \mathrm{D}$ (sessions 1 and 2 ) and 2D (session 3) imaging. The 2D imaging session was added to assess the practicability of STFR brain imaging of a thin 


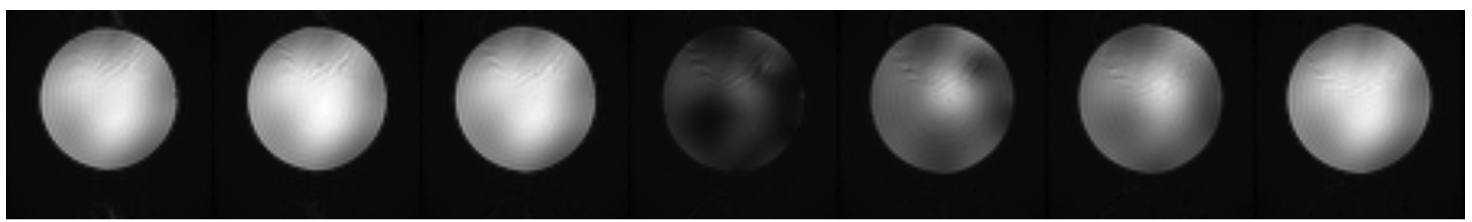

a

b

FIG. 6. Ability of the tip-up pulse to align magnetization within the imaging slice along the longitudinal axis. a: Residual transverse magnetization immediately after the tip-up pulse (time-point 2 in Fig. 1), phantom observations. Seven slices are shown. The signal in the desired imaging region (center slice) is significantly suppressed compared with neighboring slices, indicating that the tip-up pulse aligns most of the magnetization with the longitudinal axis, as desired. b: Predicted magnetization for the center slice, Bloch simulation results. Simulated and observed residual magnetization patterns are in good agreement.

2D slice, using the theoretically optimal flip angle of $16^{\circ}$ (and $32^{\circ}$ for bSSFP) for gray matter at 3T. The 3D imaging sessions used echo time $=3.7 \mathrm{~ms}, T_{\text {free }}=6.8 \mathrm{~ms}$, acquisition bandwidth $\pm 31.25 \mathrm{kHz}$, and the same tip-down pulse as in the phantom experiments (sinc, $8^{\circ}$ ). The $2 \mathrm{D}$ imaging session used echo time $=4.4 \mathrm{~ms}, T_{\text {free }}=7.4 \mathrm{~ms}$, acquisition bandwidth $\pm 31.25 \mathrm{kHz}$, and a $4 \mathrm{~mm}$ slice-selective Shinnar-Le Roux pulse of time-bandwidth product 4 (13). In the blipped acquisition (session 1), we varied the magnitude of the tip-up pulse $\beta$, to investigate its effect on image contrast. In each case, the minimum achievable TR was used, except where noted otherwise.

\section{RESULTS}

\section{Experimental Validation of Eqs. 1 and 2}

Figure 5b shows the dependence of the steady-state signal on tip-up angle $\beta$, for fixed tip-down angle $\alpha$, and for zero phase mismatch $(\Delta \theta=0)$. As the actual flip angle may deviate from the AFI measurement, we calculated three different curves, corresponding to $\alpha=13.5^{\circ}$ (the mean observed value), $14.9^{\circ}$, and $16.2^{\circ}$. The latter value, which was obtained by multiplying the measured flip angle by 1.2 , produces a reasonably good fit to the observed signal curve.

Figure 5c shows the calculated and observed steady-state signal as a function of flip angle, with $\beta=\alpha$. As the actual flip angle $\alpha$ may deviate from the AFI measurement $\alpha_{\mathrm{AFI}}$, we calculated two different experimental curves, corresponding to $\alpha=\alpha_{\text {AFI }}$ and $\alpha=1.2 \times \alpha_{\text {AFI. }}$. Measurements were made within voxels that were close to on-resonance, such that $\Delta \theta \simeq 0$. Again, the observed curve is in reasonable agreement with the theoretical curve calculated from Eq. 1, particularly for $\alpha=1.2 \times \alpha_{\mathrm{AFI}}$. We see that the acquired signal peaks at a low flip angle.

Figure $5 \mathrm{~d}$ shows the calculated and observed steady-state signal as a function of phase mismatch $\Delta \theta$, for $\beta=\alpha$. The calculation was based on the measured flip angle in each pixel (using AFI), multiplied by 1.2. Again, we generally observe good agreement between observation and theory.

Figure 5 shows that Eqs. 1 and 2 provide a reasonably accurate description of the STFR signal. For gray matter at $3 \mathrm{~T}$, these equations predict that it is necessary to achieve tip-up phase accuracy of approximately $\pm 40^{\circ}$ to avoid signal loss of more than $50 \%$.

\section{Phantom Observations}

Figure 6a shows the residual transverse magnetization immediately after the tip-up pulse (time-point " 2 " in Fig. 1). The signal in the desired imaging region (center slice) is significantly suppressed compared with neighboring slices, indicating that the tip-up pulse aligns most of the magnetization with the $z$ axis, as desired.

Figure 7 shows matched SPGR, bSSFP, and STFR images and illustrates that STFR can produce enhanced signal compared to a fully spoiled acquisition (SPGR). Note the absence of banding artifacts in the STFR image.

\section{Human Volunteer Observations, Blipped Tip-Up Pulse}

Figure 8 shows brain imaging results using SPGR, bSSFP, and the proposed STFR method. SPGR produces relatively

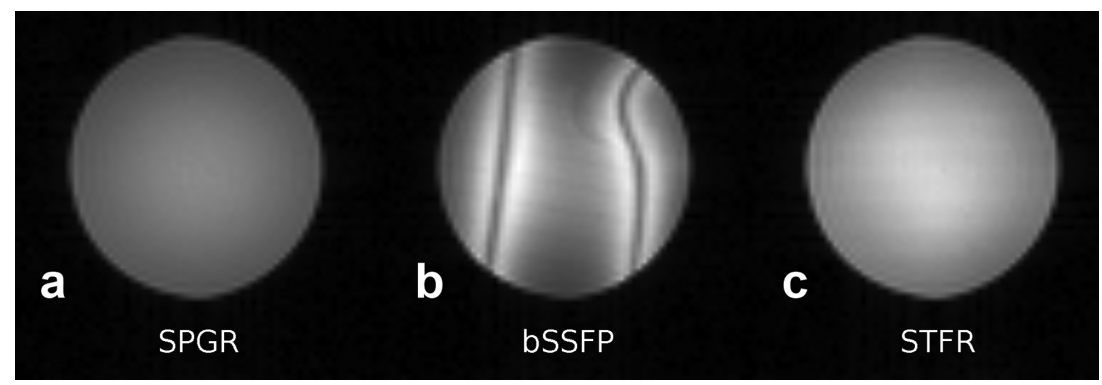

FIG. 7. Comparison of (a) SPGR, (b) bSSFP, and (c) STFR acquisitions in a uniform gel phantom. In the STFR acquisition (c), the signal was acquired at time-point 1 in Fig. 1. STFR produces higher signal than SPGR and no banding artifacts. 


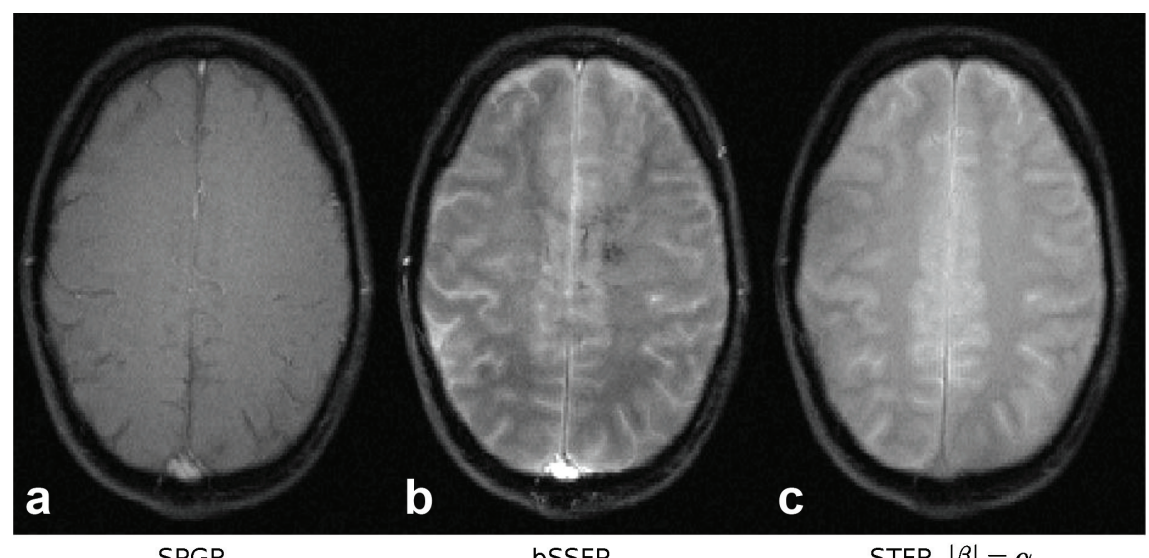

SPGR

bSSFP

STFR, $|\beta|=\alpha$

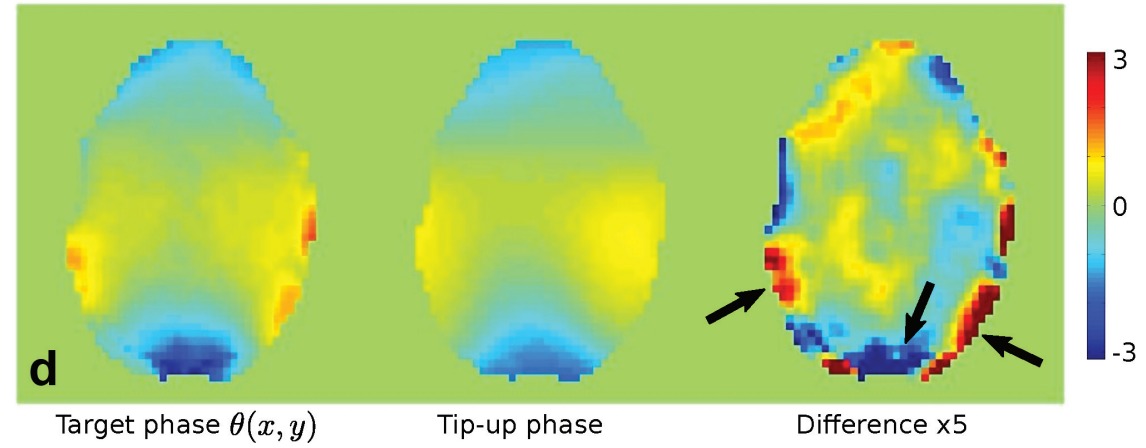

FIG. 8. Comparison of (a) SPGR (TR $=10.9 \mathrm{~ms}$ ), (b) bSSFP (TR $=9.5 \mathrm{~ms})$, and (c) the proposed STFR sequence $(|\beta|=\alpha$, TR $=11.1 \mathrm{~ms})$. The tip-down excitation pulse was the same for all acquisitions $\left(\alpha=8^{\circ}\right)$. In (c), the tip-up pulse consisted of a train of 10 blips, similar to the pulse sequence shown in Fig. 1b. SPGR (a) produces relatively low tissue signal, consistently dark CSF, and bright vessel signal due to in-flow enhancement. Balanced SSFP (b) produces bright CSF in accordance with its high $T_{2} / T_{1}$ ratio and high vessel signal. STFR produces good gray/white matter contrast similar to bSSFP, indicating that the tip-up pulse introduces $T_{2}$-weighting. d: Comparison of target phase $\theta(x, y)$ (left) and the simulated phase of the tip-up pulse (units of radians). The phase of the tip-up pulse is in good agreement with the target phase, as desired.

low tissue signal, dark CSF, and bright vessel signal due to in-flow enhancement. Balanced SSFP produces bright CSF in accordance with its high $T_{2} / T_{1}$ ratio and high vessel signal, both of which are generally undesirable. However, the gray/white matter contrast in bSSFP is excellent, which makes this sequence useful for $T_{2}$ lesion imaging, for example. The origin of the dark U-shaped band in Fig. 8b is unclear, but it may be due to aliasing from spins outside of the imaging slice that reside in the so-called bSSFP "transition band." STFR (Fig. 8c) produces excellent gray/white matter contrast similar to bSSFP, indicating that the tip-up pulse introduces $T_{2}$-weighting into the steady-state magnetization, as expected. However, we observe some signal shading across the image, in the areas indicated by the arrows in Fig. 8d. These regions generally coincide with regions that have high phase mismatch between the spin phase and the phase of the tip-up pulse $(>\pi / 5)$, as expected. Note that the phase difference in some pixels exceed $\pi / 5$ and therefore appear truncated in the phase-difference image in (c).

Figure 9 shows the effect of varying the tip-up angle $\beta$ on image contrast. For the highest value of $\beta$ ( $8^{\circ}$, Fig. $\left.9 \mathrm{~d}\right)$, the anterior CSF signal is bright, just as in bSSFP. As $\beta$ decreases, we observe that the gray and white matter signals also decrease and that the gray/white matter contrast decreases. This behavior is in qualitative agreement with theory. We also observe that the anterior CSF signal decreases relatively rapidly with decreasing $\beta$, also in accordance with theory (Fig. 9a). Finally, we observe that the vessel signal increases with decreasing $\beta$, indicating increased in-flow enhancement (19). In other words, the image contrast becomes increasingly SPGR-like with decreasing $\beta$.

\section{Human Volunteer Observations, Spiral Tip-Up Pulse}

Figure 10 shows the results of 3D STFR imaging using a 2D spiral tip-up pulse tailored to the central slice. STFR fails to recover the signal in the frontal sinus, indicating that the $B 0$ inhomogeneity is too large for this particular spiral tip-up pulse. In particular, Fig. 10c shows that the phase mismatch in the frontal sinus exceeds $\pi / 5\left(\simeq 40^{\circ}\right)$, and therefore, pixels in this region appear truncated in the phase-difference image in (c). Furthermore, the magnitude of the tip-up pulse also shows relatively large deviations in this region (not shown), which may further contribute to the signal loss. Nevertheless, most of the image exhibits good tissue contrast. However, CSF appears somewhat suppressed in the STFR image compared to bSSFP. This is unexpected, because our theory predicts relatively bright 

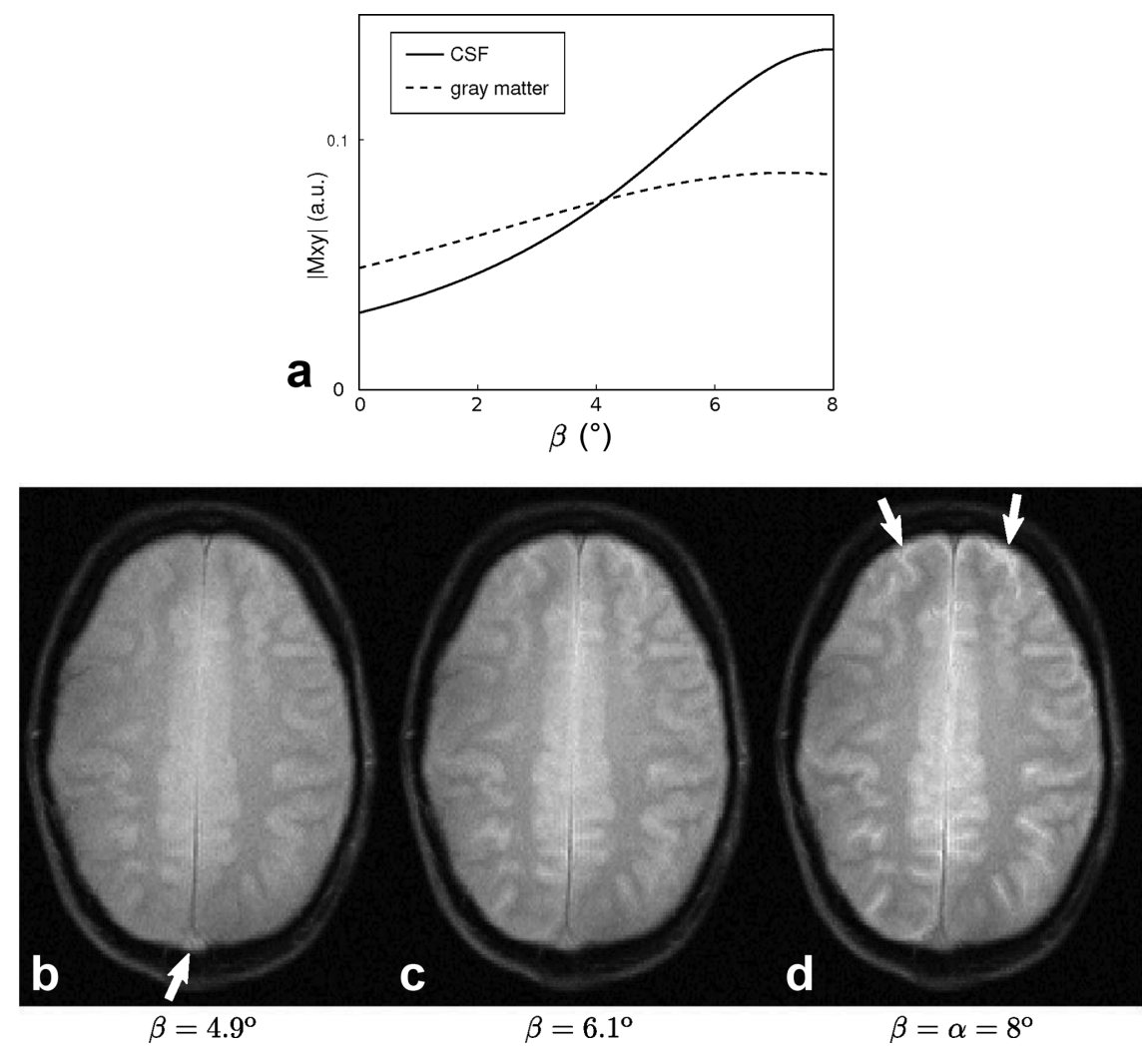

FIG. 9. Effect of varying the tip-up angle $(\beta)$ on in vivo image contrast. a: Calculated steady-state signal for CSF and gray matter at $3 \mathrm{~T}$, using Eq. $1\left(T_{\text {free }} / \mathrm{TR}=8 / 11 \mathrm{~ms}, \alpha=8^{\circ}\right)$. b-d: In vivo results, using a blipped tip-up pulse (as in Fig. $1 \mathrm{~b}$ ), with TR=11.1 ms. The same slice was imaged three times, and the amplitude of the tip-up pulse $\beta(x, y)$ was set to (b) $4.9^{\circ}$, (c) $6.1^{\circ}$, and (d) $8^{\circ}$. The tip-down pulse $\alpha$ was held fixed at $8^{\circ}$. Note the behavior of CSF (arrows in (d)) and through-plane vessel signal (arrow in (b)).

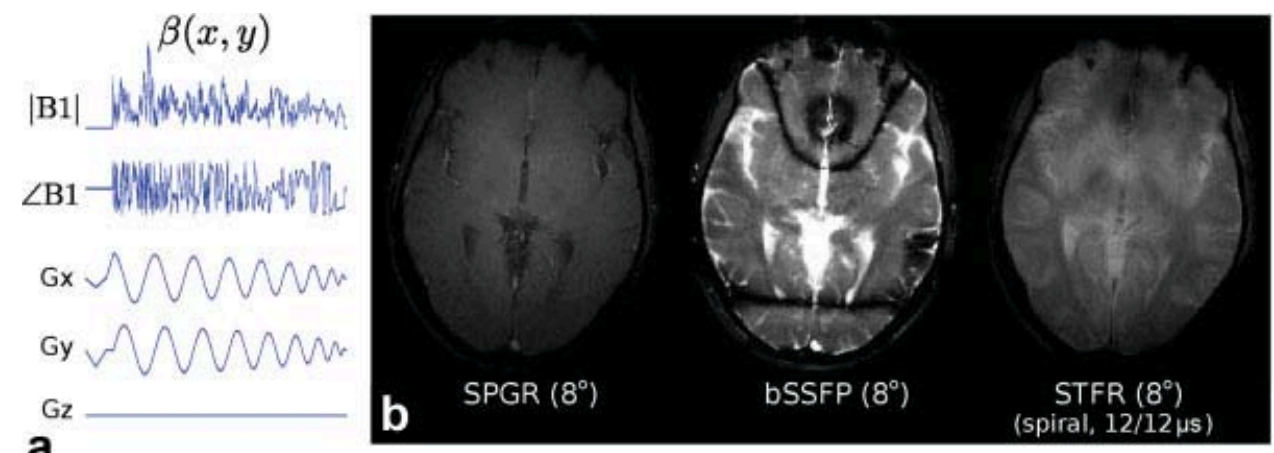

a

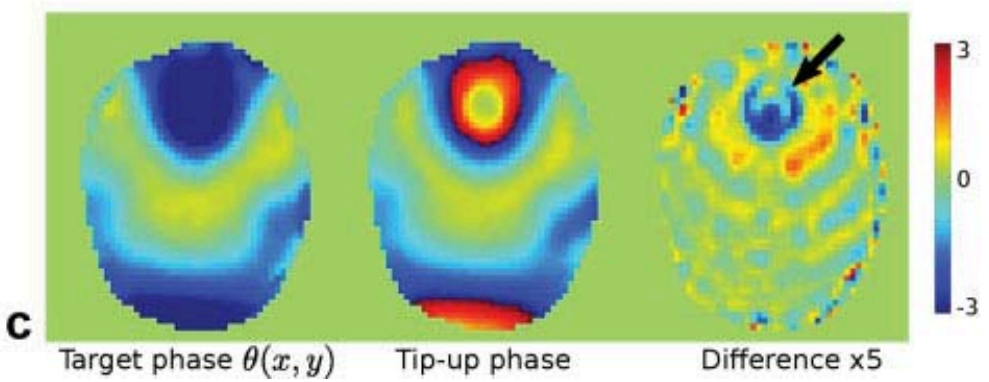

FIG. 10. Feasibility of STFR imaging using a tip-up pulse with a spiral excitation $k$-space trajectory of duration $2.3 \mathrm{~ms}$. a: Tip-up pulse sequence diagram. b: Comparison of SPGR, bSSFP, and STFR acquisitions, using the same tip-down angle $\alpha\left(8^{\circ}\right)$ and TR (11.8 ms). Note that the TR of bSSFP was slightly longer than strictly necessary, which reduces the spacing between the bands somewhat. As in Figs. 8 and 9, the middle slice from a 3D acquisition is shown. The numbers below the STFR image indicate the applied $x / y$ gradient delays in microseconds. c: The target phase $\theta(x, y)$ and the (simulated) phase of the tip-up pulse are in good agreement, except near the frontal sinus. [Color figure can be viewed in the online issue, which is available at wileyonlinelibrary.com.] 

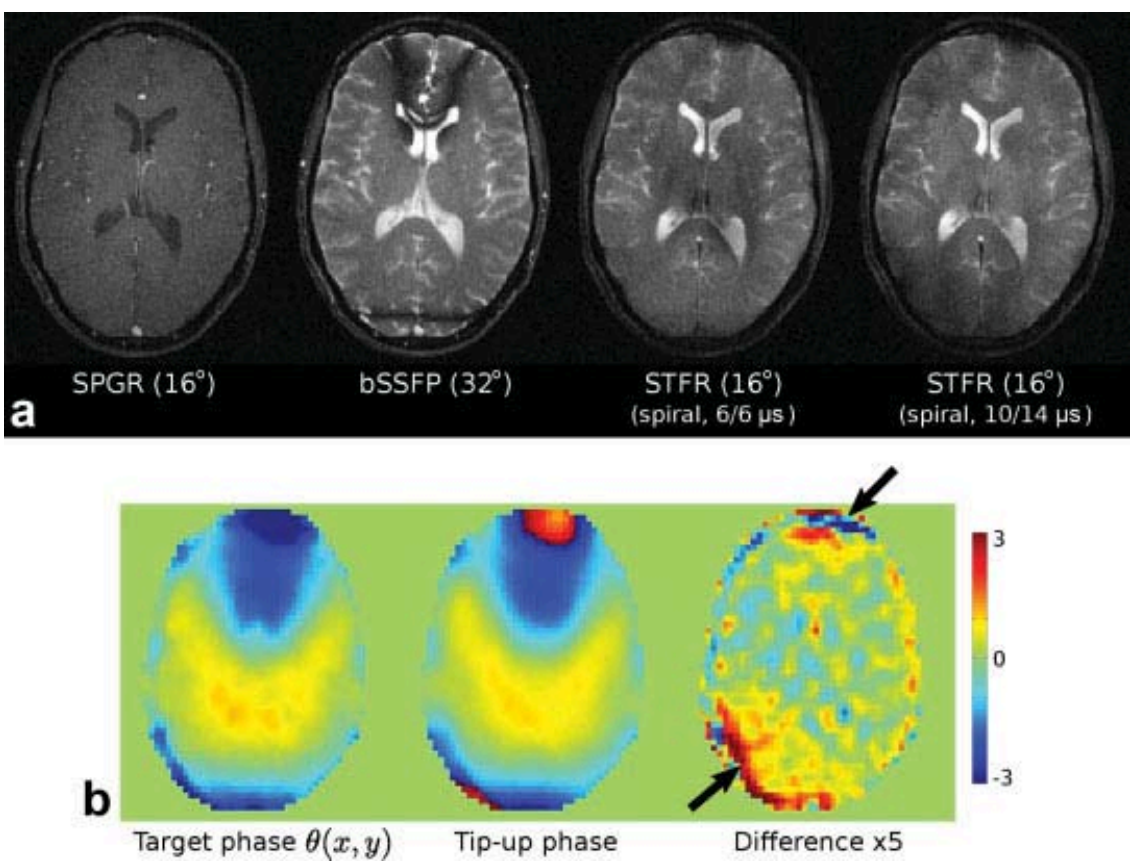

FIG. 11. Feasibility of 2D STFR imaging with a spiral tip-up pulse, using the theoretically optimal flip angle of $16^{\circ}$ for gray matter at $3 \mathrm{~T}^{\text {. The }}$ tip-up pulse was similar to that shown in Fig. $10 \mathrm{a}$. a: Comparison of SPGR (TR $=14.2 \mathrm{~ms}), \mathrm{bSSFP}(\mathrm{TR}=9.9 \mathrm{~ms})$, and STFR(TR $=14.2 \mathrm{~ms})$ acquisitions. b: The target phase $\theta(x, y)$ and the (simulated) phase of the tip-up pulse are in good agreement, except in the regions indicated by the arrows. [Color figure can be viewed in the online issue, which is available at wileyonlinelibrary.com.]

CSF for both bSSFP and STFR, independent of flip angle (Fig. 3a). The source of the low CSF signal in Fig. 10c is currently unknown but may be due to low tip-up angle $\beta$ (see, e.g., Fig. 9a).

Figure 11 shows the results of 2D STFR imaging using a similar tip-up pulse as that shown in Fig. 10a. These results are in good overall agreement with the 3D imaging results. In particular, we observe bright CSF and good gray/white matter contrast, except in regions of relatively high phase mismatch (arrows in Fig. 11b).

\section{DISCUSSION}

The proposed method relies on the ability of the tip-up pulse to align the magnetization with the longitudinal axis. Our simplified STFR signal model, expressed in Eqs. 1 and 2 , predicts that the phase of the tip-up pulse must be within a few tens of degrees to avoid significant signal loss. Our in vivo observations, using both blipped and spiral tipup pulses, confirm this prediction. In particular, we have observed that a phase mismatch of $\sim \pi / 5$, or $\sim 40^{\circ}$, produces a noticeable loss in image contrast. Generally speaking, for a given RF pulse duration and excitation $k$-space trajectory, the accuracy of the tip-up pulse decreases with increasing $B 0$ inhomogeneity. Similarly, for a given phase accrual $\theta(x, y)$ and a given excitation $k$-space trajectory, the accuracy of the tip-up pulse decreases with decreasing $R F$ pulse duration. For example, we have found that using a blipped excitation $k$-space trajectory of total duration $1.5 \mathrm{~ms}$, it is not possible to design accurate tip-up pulses in axial slices at the level of the sinuses. We anticipate that the preferred implementation of the proposed method will use spiral or other echo-planar excitation $k$-space trajectories, which offer improved performance in theory, but also introduce practical challenges such as sensitivity to eddy currents and gradient delays.

The tip-up pulse performance can be improved by reducing the free precession time $T_{\text {free }}$. In our current implementation using a $\pm 31.25 \mathrm{kHz}$ acquisition bandwidth, the total duration of the frequency-encode gradients was $\sim 6 \mathrm{~ms}$, including the defocusing and refocusing gradient lobes. Alternative readout trajectories such as partial Fourier and radial can reduce $T_{\text {free }}$ and hence improve the homogeneity of the target phase pattern used in the tip-up pulse design (Eq. 3). A similar strategy can in principle be used to reduce the TR in bSSFP, although we anticipate that bSSFP may be more sensitive to the changing eddy currents caused by a radial acquisition scheme. In addition, it may be possible to reduce the time from the peak to the end of the tip-down pulse, e.g., using VERSE RF pulse design (20).

In the future, we anticipate that the proposed implementation will benefit directly from hardware improvements in two areas: (1) parallel excitation, which refers to the use of multiple independently controlled transmission coils, and (2) high-order gradient shim systems. Parallel excitation allows improved pulse performance or reduced pulse length, in a manner analogous to parallel imaging. The goal of high-order gradient shimming is to reduce the static $B 0$ inhomogeneity, which makes the target excitation phase pattern smoother and hence easier to produce.

We have observed that CSF is suppressed when the tip-up angle $\beta$ is less than the tip-down angle $\alpha$ and throughplane vessel signal is also suppressed due to partial saturation of out-of-slice spins. These observations suggest that steady-state $T_{2}$-weighted imaging with intrinsic 
CSF suppression and lack of in-flow enhancement may be possible using the proposed sequence, which could be useful as an alternative to FLAIR spin-echo or FLAIR bSSFP imaging of $T_{2}$ lesions in, e.g., multiple sclerosis patients.

Fat resonates at a frequency offset of $-440 \mathrm{~Hz}$ compared to water protons at $3 \mathrm{~T}$, and therefore, the tip-up pulses designed from water-only $B 0$ maps will not be appropriate for fat. As a result, the signal level in local fat deposits is unpredictable and may vary spatially. It may be possible to account for fat explicitly in the RF design algorithm, with the goal of suppressing fat uniformly across the slice. Alternatively, it may be possible to incorporate a fat suppression pulse, analogous to the fat-saturated bSSFP sequence in Ref. 21.

We do not yet know whether the discrepancies between the bSSFP images and the spiral STFR acquisitions observed in Figs. 10 and 11 can be fully explained by the transverse phase mismatch, or whether other sources of error such as spiral gradient imperfections (i.e., trajectory errors during tip-up RF transmission) or imperfect spoiling also play a significant role. Further studies are needed to address this issue.

Figure 5 shows that the proposed STFR signal theory, expressed in Eqs. 1 and 2, provides a good description of the STFR signal under ideal imaging conditions. The discrepancies between theory and observation in Fig. 5 may be due to several possible sources, including nonideal slice profile, inaccurate flip angle measurements, or intrinsic errors in our signal model (e.g., due to the simplifying assumption that the tip-up pulse is instantaneous).

\section{CONCLUSIONS}

We have presented a proof-of-principle demonstration of STFR imaging with 2D tailored RF pulses, and have shown that brain imaging is feasible using a standard head coil and a short tip-up pulse ( $\sim 2 \mathrm{~ms})$. The resulting images show excellent gray/white matter contrast, and point to the possibility of rapid steady-state $T_{2} / T_{1}$-weighted imaging with intrinsic suppression of CSF, vessel signal, and off-resonance artifacts. The performance of the proposed method depends on the accuracy of the tip-up pulse, and in the future, we expect STFR imaging to benefit significantly from parallel excitation hardware and high-order gradient shim systems.

\section{REFERENCES}

1. Heilman JA, Derakhshan JJ, Riffe MJ, Gudino N, Tkach J, Flask CA, Duerk JL, Griswold MA. B0 and B1 Correction using the inherent degrees of freedom of a multi-channel transmit array. In Proceedings of the 17th Annual Meeting of ISMRM, Honolulu, Hawaii, USA, 2009. p. 251.
2. Nielsen JF, Yoon D, Noll DC. Suppression of banding and transient signal oscillations in balanced SSFP using a spoiled RF pre-phasing approach. In Proceedings of the 18th Annual Meeting of ISMRM, Stockholm, Sweden, 2010. p. 77.

3. Nielsen JF, Yoon D, Hollingsworth NA, Moody KL, McDougall MP, Wright SM, Noll DC. Joint optimization of tip-down and tip-up RF pulses in small-tip (non-spin-echo) fast recovery imaging. In Proceedings of the 19th Annual Meeting of ISMRM, Montreal, Canada, 2011. p. 209.

4. Scheffler K, Lehnhardt S. Principles and applications of balanced SSFP techniques. Eur Radiol 2003;13:2409-2418.

5. Bangerter NK, Hargreaves BA, Vasanawala SS, Pauly JM, Gold GE, Nishimura DG. Analysis of multiple-acquisition SSFP. Magn Reson Med 2004;51:1038-1047.

6. Overall WR, Nishimura DG, Hu BS. Steady-state sequence synthesis and its application to efficient fat-suppressed imaging. Magn Reson Med 2003;50:550-559.

7. Cukur T, Nishimura DG. Multiple repetition time balanced steady-state free precession imaging. Magn Reson Med 2009;62:193-204.

8. Nayak KS, Lee HL, Hargreaves BA, Hu BS. Wideband SSFP: alternating repetition time balanced steady state free precession with increased band spacing. Magn Reson Med 2007;58:931-938.

9. Leupold J, Hennig J, Scheffler K. Alternating repetition time balanced steady state free precession. Magn Reson Med 2006;55:557565.

10. Zur Y, Wood ML, Neuringer LJ. Spoiling of transverse magnetization in steady-state sequences. Magn Reson Med 1991;21:251-263.

11. Hsu JJ, Glover GH, Zaharchuk G. Optimizing saturation-recovery measurements of the longitudinal relaxation rate under time constraints. Magn Reson Med 2009;62:1202-1210.

12. Wong EC, Liu TT, Luh WM, Frank LR, Buxton RB. T1 and T2 selective method for improved SNR in CSF-attenuated imaging: T2-FLAIR. Magn Reson Med 2001;45:529-532.

13. Pauly JM, Le Roux P, Nishimura DG, Macovski A. Parameter relations for the Shinnar-Le Roux selective excitation pulse design algorithm. IEEE Trans Med Imaging 1991;10:53-65.

14. Yarnykh VL. Actual flip-angle imaging in the pulsed steady state: a method for rapid three-dimensional mapping of the transmitted radiofrequency field. Magn Reson Med 2007;57:192-200.

15. Zhang Z, Yip CY, Grissom W, Noll DC, Boada FE, Stenger VA. Reduction of transmitter B1 inhomogeneity with transmit SENSE slice-select pulses. Magn Reson Med 2007;57:842-847.

16. Pauly JM, Nishimura DG, Macovski A. A k-space analysis of small tip excitation. J Magn Reson 1989;81:43-56.

17. Yoon D, Fessler JA, Gilbert AC, Noll DC. Simultaneous signal loss correction from B1 and B0 inhomogeneity in BOLD fMRI with parallel excitation. In Proceedings of ISMRM 3rd International Workshop on Parallel MRI, Santa Cruz, California, USA, 2009. p. 38.

18. Yip CY, Fessler JA, Noll DC. Iterative RF pulse design for multidimensional, small-tip-angle selective excitation. Magn Reson Med 2005;54:908-917.

19. Han M, Hargreaves BA. Reduction of flow artifacts by using partial saturation in RF-spoiled gradient-echo imaging. Magn Reson Med 2011;65:1326-1334.

20. Hargreaves BA, Cunningham CH, Nishimura DG, Conolly SM. Timeoptimal VERSE excitation for $3 \mathrm{~d}$ balanced SSFP imaging. In Proceedings of the 12th Annual Meeting of ISMRM, Kyoto, Japan, 2004. p. 260.

21. Scheffler K, Heid O, Hennig J. Magnetization preparation during the steady state: fat-saturated 3D trueFISP. Magn Reson Med 2001;45:10751080 . 